\title{
Implicit Difference Methods for Quasilinear Differential Functional Equations on the Haar Pyramid
}

\author{
A. Kepczyńska
}

\begin{abstract}
We present a new class of numerical methods for quasilinear first order partial functional differential equations. The numerical methods are difference schemes which are implicit with respect to the time variable. The existence of approximate solutions is proved by using a theorem on difference inequalities. The proof of the stability is based on a comparison technique with nonlinear estimates of the Perron type for given operators. Numerical experiments are presented. Results obtained in this paper can be applied to differential integral problems and to equations with deviated variables.
\end{abstract}

Keywords. Initial problems on the Haar pyramid, implicit difference methods, stability and convergence, interpolating operators

Mathematics Subject Classification (2000). 65M10, 65M15, 35R10

\section{Introduction}

For any metric spaces $X$ and $Y$ we denote by $C(X, Y)$ the class of all continuous functions from $X$ to $Y$. We will use vectorial inequalities with the understanding that the same inequalities hold between their corresponding components.

Let $E$ be the Haar pyramid

$$
E=\left\{(t, x)=\left(t, x_{1}, \ldots, x_{n}\right) \in \mathbb{R}^{1+n}: t \in[0, a], x \in[-b+M t, b-M t]\right\},
$$

where $a>0, b=\left(b_{1}, \ldots, b_{n}\right), M=\left(M_{1}, \ldots, M_{n}\right) \in \mathbb{R}_{+}^{n}, \mathbb{R}_{+}=[0,+\infty)$, and $b>M a$. Write

$$
E_{0}=\left[-d_{0}, 0\right] \times[-d, d], \quad E_{t}=\left(E_{0} \cup E\right) \cap\left(\left[-d_{0}, t\right] \times \mathbb{R}^{n}\right), \quad 0<t \leqslant a,
$$

A. Kępczyńska: Faculty of Applied Physics and Mathematics, Gdańsk University of Technology, G. Narutowicz Street 11/12, 80-226 Gdańsk, Poland; annak@mif.pg.gda.pl 
where $d_{0} \in \mathbb{R}_{+}$. Put $\Omega=E \times C\left(E_{0} \cup E, \mathbb{R}\right)$ and suppose that $f: \Omega \rightarrow \mathbb{R}^{n}, f=$ $\left(f_{1}, \ldots, f_{n}\right), g: \Omega \rightarrow \mathbb{R}, \varphi: E_{0} \rightarrow \mathbb{R}$ are given functions. We consider the differential functional equation

$$
\partial_{t} z(t, x)=\sum_{i=1}^{n} f_{i}(t, x, z) \partial_{x_{i}} z(t, x)+g(t, x, z),
$$

with the initial condition

$$
z(t, x)=\varphi(t, x) \quad \text { for }(t, x) \in E_{0} .
$$

A function $v: E_{0} \cup E \rightarrow \mathbb{R}$ is a classical solution of above problem if

(i) $v \in C\left(E_{0} \cup E, \mathbb{R}\right)$ and $v$ is of class $C^{1}$ on $E$;

(ii) $v$ satisfies (1) on $E$ and initial condition (2) holds.

The function $f: \Omega \rightarrow \mathbb{R}^{n}$ is said to satisfy the Volterra condition if for each $(t, x) \in E$ and for $z, \bar{z} \in C\left(E_{0} \cup E, \mathbb{R}\right)$ such that $z(\tau, y)=\bar{z}(\tau, y)$ for $(\tau, y) \in E_{t}$ we have $f(t, x, z)=f(t, x, \bar{z})$. Note that the Volterra condition for $f$ means that the value of $f$ at the point $(t, x, z)$ of the space $\Omega$ depends on $(t, x)$ and on the restriction of $z$ to the set $E_{t}$. In the same way we define the Volterra condition for the function $g$. In the present paper we assume that $f$ and $g$ satisfy the Volterra condition and we consider classical solutions of (1), (2).

The Haar pyramid is a natural set for the existence and uniqueness of initial problems for differential and functional differential equations (see $[8,12,15,16]$ ). We are interested in the construction of a method for the approximation of solutions to problem (1), (2) with solutions of difference functional equations and in an estimation of the difference between these solutions.

In recent years, a number of papers concerning difference methods for functional differential equations have been published. It is easy to construct an explicit difference method for a nonlinear differential equation which satisfies the consistency conditions on all sufficiently regular solutions of a considered problem. The main task in these investigations is to find a finite difference equation which is stable. The method of difference inequalities or simple theorems on recurrent inequalities are used in the investigation of the stability of difference functional problems.

Convergence results are also based on a general theorem on an error estimate of approximate solutions to functional difference equations of the Volterra type with initial or initial boundary conditions and with an unknown function of several variables.

The problems mentioned above have an extensive bibliography. It is not our aim to show a full review of papers concerning numerical methods for partial functional differential equations. We shall mention only those which contain such review; they are $[2,3,6,7,13,14]$ and the monograph [8]. The papers [9-11] 
initiated the investigations of implicit difference methods for first order partial differential equations.

The numerical method of lines for nonlinear functional differential equations was considered in [1]. By using a discretization in spatial variables, the nonlinear equations are replaced by sequences of initial problems for ordinary functional differential equations. The question of under what conditions the solutions of ordinary equations tend to solutions of original problems is investigated. The proofs of the convergence of the numerical methods of lines are based on differential inequalities technique.

In the paper we present another approach to the difference methods for problem (1), (2). We prove that there is a class of implicit difference methods for (1), (2) which are convergent. The stability of difference schemes is investigated by using a comparison method with nonlinear estimates of the Perron type for given functions.

The paper is organized as follows. In Section 2 we propose an implicit difference method of the Euler type for problem (1), (2). This leads to an implicit difference functional problem. The existence and uniqueness of solutions of such problems are considered in Section 3. The method of difference inequalities is used. The next section deals with a theorem on the error estimate for approximate solutions of implicit difference functional equations. A convergence result and an error estimate are presented in Section 4. In the next section we discuss the problem of the existence of solutions of implicit difference schemes on rectangular domain. More precisely, we show that there is a class of initial problems (1), (2) for which approximate solutions can be calculated easily. Numerical examples are given in the last part of the paper.

Note that differential equations with deviated variables and differential integral equations can be obtained from (1) by specializing the operators $f$ and $g$. Existence result for problem (1), (2) are given in [8, Chapter 2].

\section{Discretization of quasilinear equations}

We will denote by $F(X, Y)$ the class of all functions defined on $X$ and taking values in $Y$ where $X$ and $Y$ are arbitrary sets. For $x, y \in \mathbb{R}^{n}, x=\left(x_{1}, \ldots, x_{n}\right)$, $y=\left(y_{1}, \ldots, y_{n}\right)$ we write $\|x\|=\sum_{i=1}^{n}\left|x_{i}\right|$ and $x \diamond y=\left(x_{1} y_{1}, \ldots, x_{n} y_{n}\right)$. For a function $z \in C\left(E_{0} \cup E, \mathbb{R}\right)$ and for a point $t \in[0, a]$ we put

$$
\|z\|_{t}=\max \left\{|z(\tau, x)|:(\tau, x) \in E_{t}\right\} .
$$

We define a mesh on the set $E_{0} \cup E$ in the following way. Let $\left(h_{0}, h^{\prime}\right)$, $h^{\prime}=\left(h_{1}, \ldots, h_{n}\right)$, stand for steps of the mesh. Let us denote by $H$ the set of all $h=\left(h_{0}, h^{\prime}\right)$ such that there are $K_{0} \in \mathbb{N}$ and $K=\left(K_{1}, \ldots, K_{n}\right) \in \mathbb{N}^{n}$ with the 
properties $K_{0} h_{0}=d_{0}$ and $K \diamond h^{\prime}=b$. For $h \in H$ and for $(r, m) \in \mathbb{Z}^{1+n}$, where $m=\left(m_{1}, \ldots, m_{n}\right)$, we define nodal points as follows:

$$
t^{(r)}=r h_{0}, \quad x^{(m)}=m \diamond h^{\prime}, \quad x^{(m)}=\left(x_{1}^{\left(m_{1}\right)}, \ldots, x_{n}^{\left(m_{n}\right)}\right) .
$$

Let $N_{0} \in \mathbb{N}$ be defined by the relations $N_{0} h_{0} \leqslant a<\left(N_{0}+1\right) h_{0}$. Write

$$
R_{h}^{1+n}=\left\{\left(t^{(r)}, x^{(m)}\right):(r, m) \in \mathbb{Z}^{1+n}\right\}
$$

and

$$
\begin{gathered}
E_{h}=E \cap R_{h}^{1+n}, \quad E_{h .0}=E_{0} \cap R_{h}^{1+n}, \\
E_{h . r}=\left(E_{h .0} \cup E_{h}\right) \cap\left(\left[-d_{0}, t^{(r)}\right] \times \mathbb{R}^{n}\right), \quad 0 \leqslant r \leqslant N_{0} .
\end{gathered}
$$

Moreover we put

$$
\begin{aligned}
E_{h}^{\prime} & =\left\{\left(t^{(r)}, x^{(m)}\right) \in E_{h}:\left(t^{(r+1)}, x^{(m)}\right) \in E_{h}\right\}, \\
I_{h} & =\left\{t^{(r)}: 0 \leqslant r \leqslant N_{0}\right\}, \quad I_{h}^{\prime}=I_{h} \backslash\left\{t^{\left(N_{0}\right)}\right\} .
\end{aligned}
$$

For a function $z: E_{h .0} \cup E_{h} \rightarrow \mathbb{R}$ we write $z^{(r, m)}=z\left(t^{(r)}, x^{(m)}\right)$ and

$$
\|z\|_{h . i}=\max \left\{\left|z^{(r, m)}\right|:\left(t^{(r)}, x^{(m)}\right) \in E_{h . i}\right\}
$$

where $0 \leqslant i \leqslant N_{0}$. Let $e_{i}=(0, \ldots, 0,1,0, \ldots, 0) \in \mathbb{R}^{n}, 1 \leqslant i \leqslant n$, where 1 is the $i$-th coordinate.

Classical difference methods for $(1),(2)$ consist in replacing partial derivatives $\partial_{t}$ and $\left(\partial_{x_{1}}, \ldots, \partial_{x_{n}}\right)=\partial_{x}$ with difference operators $\delta_{0}$ and $\left(\delta_{1}, \ldots, \delta_{n}\right)=\delta$, respectively. Moreover, because equation (1) contains the functional variable we need an interpolating operator $T_{h}: F\left(E_{h .0} \cup E_{h}, \mathbb{R}\right) \rightarrow C\left(E_{0} \cup E, \mathbb{R}\right)$. This leads to the difference equation

$$
\delta_{0} z^{(r, m)}=\sum_{i=1}^{n} f_{i}\left(t^{(r)}, x^{(m)}, T_{h}[z]\right) \delta_{i} z^{(r, m)}+g\left(t^{(r)}, x^{(m)}, T_{h}[z]\right)
$$

with the initial condition

$$
z^{(r, m)}=\varphi_{h}^{(r, m)} \quad \text { on } E_{h .0},
$$

where $\varphi_{h}: E_{h .0} \rightarrow \mathbb{R}$ is a given function. The following examples of equations (3) are considered in the literature. Write

$$
\delta_{0} z^{(r, m)}=\frac{1}{h_{0}}\left[z^{(r+1, m)}-z^{(r, m)}\right]
$$

and

$$
\begin{array}{ll}
\delta_{j} z^{(r, m)}=\frac{1}{h_{j}}\left[z^{\left(r, m+e_{j}\right)}-z^{(r, m)}\right] & \text { for } 1 \leqslant j \leqslant \kappa \\
\delta_{j} z^{(r, m)}=\frac{1}{h_{j}}\left[z^{(r, m)}-z^{\left(r, m-e_{j}\right)}\right] & \text { for } \kappa+1 \leqslant j \leqslant n
\end{array}
$$


where $0 \leqslant \kappa \leqslant n$ is fixed. The numerical method (3), (4) with the above given $\delta_{0}$ and $\delta$ is known as the Euler method. The Lax difference scheme is the second important example. It is obtained by putting

$$
\begin{aligned}
& \delta_{0} z^{(r, m)}=\frac{1}{h_{0}}\left[z^{(r+1, m)}-\frac{1}{2 n} \sum_{i=1}^{n}\left(z^{\left(r, m+e_{i}\right)}+z^{\left(r, m-e_{i}\right)}\right)\right] \\
& \delta_{j} z^{(r, m)}=\frac{1}{2 h_{j}}\left[z^{\left(r, m+e_{j}\right)}-z^{\left(r, m-e_{j}\right)}\right], \quad 1 \leqslant j \leqslant n .
\end{aligned}
$$

The stability of difference equations generated by first order partial functional differential equations is strictly connected with the so-called CourantFriedrichs-Levy (CFL) conditions, see [4]. The (CFL) condition for equation (1) and for the Euler difference method has the form

$$
1-h_{0} \sum_{j=1}^{n} \frac{1}{h_{j}}\left|f_{j}(t, x, z)\right| \geqslant 0 \quad \text { on } \Omega_{h} .
$$

The (CFL) condition for the Lax scheme has the form

$$
1-n \frac{h_{0}}{h_{j}}\left|f_{j}(t, x, z)\right| \geqslant 0, \quad 1 \leqslant j \leqslant n, \quad \text { on } \Omega_{h} .
$$

Regularity assumptions in stability theorems for $f$ and $g$ with respect to the functional variable are the same for the both above difference methods. It is assumed that $f$ and $g$ satisfy the Lipschitz condition with respect to the functional variable. Nonlinear estimates of the Perron type for $f$ and $g$ with respect to the functional variable can be also adopted.

Suppose that the functions $f$ and $g$ are bounded on $\Omega$. The (CFL) conditions (6) and (7) state that there are requirements for the steps $h_{0}$ and $h^{\prime}=\left(h_{1}, \ldots, h_{n}\right)$ in convergence theorems for $(3),(4)$.

In the paper we present a new approach to the numerical solving of (1), (2). We prove that under natural assumptions on given functions and on the mesh there is a class of implicit difference schemes for (1), (2) which is convergent. The aim of the paper is to show that there are difference methods for (1), (2) for which the (CFL) conditions can be omitted.

We formulate the implicit difference method of the Euler type for (1), (2). Put $\Omega_{h}=E_{h}^{\prime} \times F\left(E_{h .0} \cup E_{h}, \mathbb{R}\right)$. Suppose that functions $f_{h}: \Omega_{h} \rightarrow \mathbb{R}^{n}, f_{h}=$ $\left(f_{h .1}, \ldots, f_{h . n}\right), g_{h}: \Omega_{h} \rightarrow \mathbb{R}, \varphi_{h}: E_{h .0} \rightarrow \mathbb{R}$ are given. We will approximate classical solutions of problem (1), (2) by means of solutions of the implicit difference problem

$$
\begin{aligned}
\delta_{0} z^{(r, m)} & =\sum_{i=1}^{n} f_{h . i}\left(t^{(r)}, x^{(m)}, z\right) \delta_{i} z^{(r+1, m)}+g_{h}\left(t^{(r)}, x^{(m)}, z\right) \\
z^{(r, m)} & =\varphi_{h}^{(r, m)} \quad \text { on } E_{h .0} .
\end{aligned}
$$


It is important in our considerations that the operator $\delta z=\left(\delta_{1}, \ldots, \delta_{n}\right)$ appears in (8) at the point $\left(t^{(r+1)}, x^{(m)}\right)$. The function $f_{h}$ is said to satisfy the Volterra condition if for each $\left(t^{(r)}, x^{(m)}\right) \in E_{h}^{\prime}$ and for $z, \bar{z} \in F\left(E_{h .0} \cup E_{h}, \mathbb{R}\right)$ such that $\left.z\right|_{E_{h . r}}=\left.\bar{z}\right|_{E_{h . r}}$ we have $f\left(t^{(r)}, x^{(m)}, z\right)=f\left(t^{(r)}, x^{(m)}, \bar{z}\right)$. In the same way we formulate the Volterra condition for $g_{h}$.

The difference operators $\delta_{0}, \delta=\left(\delta_{1}, \ldots, \delta_{n}\right)$ are defined in the following way:

$$
\begin{aligned}
& \delta_{0} z^{(r, m)}=\frac{1}{h_{0}}\left(z^{(r+1, m)}-z^{(r, m)}\right) \\
& \delta_{i} z^{(r+1, m)}=\frac{1}{h_{i}}\left(z^{\left(r+1, m+e_{i}\right)}-z^{(r+1, m)}\right) \quad \text { if } f_{h . i}\left(t^{(r)}, x^{(m)}, z^{(r, m)}\right) \geqslant 0 \\
& \delta_{i} z^{(r+1, m)}=\frac{1}{h_{i}}\left(z^{(r+1, m)}-z^{\left(r+1, m-e_{i}\right)}\right) \quad \text { if } f_{h . i}\left(t^{(r)}, x^{(m)}, z^{(r, m)}\right)<0 .
\end{aligned}
$$

The corresponding explicit difference scheme has the form

$$
\delta_{0} z^{(r, m)}=\sum_{i=1}^{n} f_{h . i}\left(t^{(r)}, x^{(m)}, z\right) \delta_{i} z^{(r, m)}+g_{h}\left(t^{(r)}, x^{(m)}, z\right) .
$$

If $f_{h}$ and $g_{h}$ satisfy the Volterra condition, then it is clear that there exists exactly one solution of equation (13) with initial condition (9).

We prove that under natural assumptions on the functions $f_{h}$ and $g_{h}$ there exists exactly one solution $u_{h}: E_{h .0} \cup E_{h} \rightarrow \mathbb{R}$ of problem (8), (9) with difference operators defined by (10)-(12). Let

$$
\Delta^{(r)}=\left\{x^{(m)}: x^{(m)} \in\left[-b+M t^{(r)}, b-M t^{(r)}\right]\right\}, \quad 0 \leqslant r \leqslant N_{0} .
$$

Write

$$
\begin{aligned}
& E_{h . i}^{+}[\varepsilon]=\left\{\left(t^{(r)}, x^{(m)}\right) \in E_{h}^{\prime}: b_{i}-M_{i} t^{(r)}-\varepsilon \leqslant x_{i}^{\left(m_{i}\right)} \leqslant b_{i}-M_{i} t^{(r)}\right\} \\
& E_{h . i}^{-}[\varepsilon]=\left\{\left(t^{(r)}, x^{(m)}\right) \in E_{h}^{\prime}:-b_{i}+M_{i} t^{(r)} \leqslant x_{i}^{\left(m_{i}\right)} \leqslant-b_{i}+M_{i} t^{(r)}+\varepsilon\right\},
\end{aligned}
$$

where $1 \leqslant i \leqslant n$ and $\varepsilon>0$ is such that $\varepsilon<b_{i}-M_{i} a$ for $1 \leqslant i \leqslant n$.

Assumption $H\left[f_{h}\right]$. Suppose that the function $f_{h}: \Omega_{h} \rightarrow \mathbb{R}^{n}$ satisfies the Volterra condition and

1) $h^{\prime} \leqslant M h_{0}$

2) there exists $\varepsilon>0$ such that

$$
\begin{array}{ll}
f_{h . i}\left(t^{(r)}, x^{(m)}, z\right) \leqslant 0 & \text { for }\left(t^{(r)}, x^{(m)}, z\right) \in E_{h . i}^{+}[\varepsilon] \times F\left(E_{h .0} \cup E_{h}, \mathbb{R}\right) \\
f_{h . i}\left(t^{(r)}, x^{(m)}, z\right) \geqslant 0 & \text { for }\left(t^{(r)}, x^{(m)}, z\right) \in E_{h . i}^{-}[\varepsilon] \times F\left(E_{h .0} \cup E_{h}, \mathbb{R}\right) .
\end{array}
$$


Remark 2.1. Comments on the condition 2) of Assumption $H\left[f_{h}\right]$ are given in Section 4 for $f_{h}$ defined by

$$
f_{h}(t, x, z)=f\left(t, x, T_{h}[z]\right), \quad(t, x, z) \in \Omega_{h},
$$

where $T_{h}: F\left(E_{h .0} \cup E_{h}, \mathbb{R}\right) \rightarrow C\left(E_{0} \cup E, \mathbb{R}\right)$ is an interpolating operator.

We will need the following lemma on difference inequalities generated by problem (8), (9).

Lemma 2.2. Suppose that Assumption $H\left[f_{h}\right]$ is satisfied and $h \in H, f_{h}: \Omega_{h} \rightarrow$ $\mathbb{R}^{n}, z_{h}: E_{h .0} \cup E_{h} \rightarrow \mathbb{R}$.

(I) If $z_{h}$ satisfies the implicit difference inequality

$$
z_{h}^{(r+1, m)} \leqslant h_{0} \sum_{i=1}^{n} f_{h . i}\left(t^{(r)}, x^{(m)}, z_{h}\right) \delta_{i} z_{h}^{(r+1, m)} \text { on } E_{h}^{\prime}
$$

and $z_{h}^{(r, m)} \leqslant 0$ on $E_{h .0}$, then $z^{(r+1, m)} \leqslant 0$ on $E_{h}$.

(II) If $z_{h}$ satisfies the implicit difference inequality

$$
z_{h}^{(r+1, m)} \geqslant h_{0} \sum_{i=1}^{n} f_{h . i}\left(t^{(r)}, x^{(m)}, z_{h}\right) \delta_{i} z_{h}^{(r+1, m)} \text { on } E_{h}^{\prime}
$$

and $z_{h}^{(r, m)} \geqslant 0$ on $E_{h .0}$, then $z^{(r+1, m)} \geqslant 0$ on $E_{h}$.

Proof. Consider the case (I). Suppose that $0 \leqslant r \leqslant K-1$ is fixed and $z_{h}^{(j, m)} \leqslant 0$ on $E_{h . r}$ and there exists $x^{(\widetilde{m})} \in \Delta^{(r+1)}$ such that

$$
z_{h}^{(r+1, \tilde{m})}=\max \left\{z_{h}^{(r+1, m)}:-b+M t^{(r+1)} \leqslant x^{(m)} \leqslant b-M t^{(r+1)}\right\}
$$

and

$$
z_{h}^{(r+1, \widetilde{m})}>0
$$

Write

$$
\begin{aligned}
& J_{+}^{(r, m)}\left[z_{h}\right]=\left\{i: 1 \leqslant i \leqslant n \text { and } f_{h . i}\left(t^{(r)}, x^{(m)}, z_{h}\right) \geqslant 0\right\} \\
& J_{-}^{(r, m)}\left[z_{h}\right]=\{1, \ldots, n\} \backslash J_{+}^{(r, m)}\left[z_{h}\right] .
\end{aligned}
$$

It follows from (14) that

$$
\begin{aligned}
z_{h}^{(r+1, \widetilde{m})} \leqslant & h_{0} \sum_{i \in J_{+}^{(r, \widetilde{m})}\left[z_{h}\right]} \frac{1}{h_{i}} f_{h . i}\left(t^{(r)}, x^{(\widetilde{m})}, z_{h}\right)\left[z_{h}^{\left(r+1, \widetilde{m}+e_{i}\right)}-z_{h}^{(r+1, \widetilde{m})}\right] \\
& +h_{0} \sum_{i \in J_{-}^{(r, \widetilde{m})}\left[z_{h}\right]} \frac{1}{h_{i}} f_{h . i}\left(t^{(r)}, x^{(\widetilde{m})}, z_{h}\right)\left[z_{h}^{(r+1, \widetilde{m})}-z_{h}^{\left(r+1, \widetilde{m}-e_{i}\right)}\right] \leqslant 0 .
\end{aligned}
$$

We thus get $z_{h}^{(r+1, \tilde{m})} \leqslant 0$ which contradicts (16). Then assertion (14) follows by induction with respect to $r$. In a similar way we prove that (15) holds in the case (II). This completes the proof. 
Lemma 2.3. Suppose that Assumption $H\left[f_{h}\right]$ is satisfied and $h \in H, f_{h}: \Omega_{h} \rightarrow$ $\mathbb{R}^{n}, g_{h}: \Omega_{h} \rightarrow \mathbb{R}$. Then difference functional problem (8), (9) with $\delta_{0}$ and $\delta$ defined by (10)-(12) has exactly one solution $z_{h}: E_{h .0} \cup E_{h} \rightarrow \mathbb{R}$.

Proof. Suppose that $0 \leqslant r \leqslant N_{0}-1$ is fixed and $z_{h}$ is known on the set $E_{h . r}$. Let $\left(t^{(r)}, x^{(m)}\right) \in E_{h}^{\prime}$ and $f_{h . i}\left(t^{(r)}, x^{(m)}, z^{(r, m)}\right) \geqslant 0$. Then

$$
\delta_{i} z^{(r+1, m)}=\frac{1}{h_{i}}\left[z^{\left(r+1, m+e_{i}\right)}-z^{(r+1, m)}\right] .
$$

It follows from Assumption $H\left[f_{h}\right]$ that $x^{(m)} \in \Delta^{(r+1)}$ and the difference expression $\delta_{i} z^{(r+1, m)}$ is well defined. The same conclusion can be drawn in case when $f_{h . i}\left(t^{(r)}, x^{(m)}, z\right)<0$.

The homogeneous problem corresponding to (8), (9) has the form

$$
\begin{aligned}
& z^{(r+1, m)}=h_{0} \sum_{i=1}^{n} f_{h . i}\left(t^{(r)}, x^{(m)}, z^{(r, m)}\right) \delta_{i} z^{(r+1, m)} \\
& z^{(r+1, m)}=0 \quad \text { on } E_{h .0} .
\end{aligned}
$$

It follows from Lemma 2.2 that the initial problem (19), (20) has exactly one zero solution. Therefore the problem (8), (9) has exactly one solution $z_{h}^{(r+1, m)}$, $x^{(m)} \in \Delta^{(r+1)}$ with any choice of the function $g_{h}: \Omega_{h} \rightarrow \mathbb{R}$. Consequently the function $z_{h}$ is defined and it is unique on $E_{h . r+1}$. Since $z_{h}$ is given on $E_{h .0}$ the proof is completed by induction.

\section{Approximate solutions of functional difference equations}

We start with a theorem on the error estimate of approximate solutions for implicit difference functional equations. Let us denote by $F_{h}$ the Niemycki operator corresponding to (8), i.e.,

$$
F_{h}[z]^{(r, m)}=\sum_{i=1}^{n} f_{h . i}\left(t^{(r)}, x^{(m)}, z\right) \delta_{i} z^{(r+1, m)}+g_{h}\left(t^{(r)}, x^{(m)}, z\right) .
$$

Then we consider the difference functional equation

$$
\delta_{0} z^{(r, m)}=F_{h}[z]^{(r, m)}
$$

with initial boundary condition (9). Suppose that $v_{h}: E_{h .0} \cup E_{h} \rightarrow \mathbb{R}$ and $\gamma, \alpha_{0}: H \rightarrow \mathbb{R}_{+}$are such functions that

$$
\begin{aligned}
\left|\delta_{0} v_{h}^{(r, m)}-F_{h}\left[v_{h}\right]^{(r, m)}\right| \leqslant \gamma(h) & \text { on } E_{h}^{\prime} \\
\left|\varphi_{h}^{(r, m)}-v_{h}^{(r, m)}\right| \leqslant \alpha_{0}(h) & \text { on } E_{h .0}
\end{aligned}
$$

and

$$
\lim _{h \rightarrow 0} \gamma(h)=0, \quad \lim _{h \rightarrow 0} \alpha_{0}(h)=0 .
$$


The function $v_{h}$ satisfying the above relations can be considered as an approximate solution of problem (9), (21).

Assumption $H\left[\sigma_{h}\right]$. The function $\sigma_{h}: I_{h}^{\prime} \times \mathbb{R}_{+} \rightarrow \mathbb{R}_{+}$satisfies the following conditions:

1) $\sigma_{h}(t, \cdot)$ is continuous and nondecreasing for each $t \in I_{h}^{\prime}$,

2) $\sigma_{h}(t, 0)=0$ for $t \in I_{h}^{\prime}$, and for each $\bar{c} \geqslant 1$ the difference problem

$$
\begin{aligned}
\eta^{(r+1)} & =\eta^{(r)}+h_{0} \bar{c} \sigma_{h}\left(t^{(r)}, \eta^{(r)}\right), \quad 0<r \leqslant N_{0}-1 \\
\eta^{(0)} & =0
\end{aligned}
$$

is stable in the following sense: if $\gamma, \alpha_{0}: H \rightarrow \mathbb{R}_{+}$are such functions that $\lim _{h \rightarrow 0} \gamma(h)=0, \lim _{h \rightarrow 0} \alpha_{0}(h)=0$ and $\eta_{h}: I_{h} \rightarrow \mathbb{R}_{+}$is a solution of the problem

$$
\begin{aligned}
\eta^{(r+1)} & =\eta^{(r)}+h_{0} \bar{c} \sigma_{h}\left(t^{(r)}, \eta^{(r)}\right)+h_{0} \gamma(h), \quad 0<r \leqslant N_{0}-1 \\
\eta^{(0)} & =\alpha_{0}(h)
\end{aligned}
$$

then there exists a function $\beta: H \rightarrow \mathbb{R}_{+}$such that $\eta_{h}^{(r)} \leqslant \beta(h)$ for $0 \leqslant r \leqslant N_{0}$ and $\lim _{h \rightarrow 0} \beta(h)=0$.

Assumption $H\left[f_{h}, g_{h}\right]$. Assumption $H\left[f_{h}\right]$ holds true and there is a function $\sigma_{h}: I_{h}^{\prime} \times \mathbb{R}_{+} \rightarrow \mathbb{R}_{+}$satisfying Assumption $H\left[\sigma_{h}\right]$ and such that

$$
\begin{array}{r}
\left\|f_{h}\left(t^{(r)}, x^{(m)}, z\right)-f_{h}\left(t^{(r)}, x^{(m)}, \bar{z}\right)\right\| \leqslant \sigma_{h}\left(t,\|z-\bar{z}\|_{h . r}\right) \\
\left|g_{h}\left(t^{(r)}, x^{(m)}, z\right)-g_{h}\left(t^{(r)}, x^{(m)}, \bar{z}\right)\right| \leqslant \sigma_{h}\left(t,\|z-\bar{z}\|_{h . r}\right)
\end{array}
$$

on $E_{h .0} \cup E_{h}$.

Remark 3.1. It follows from (29), (30) that $f_{h}$ and $g_{h}$ satisfy the Volterra condition.

Theorem 3.2. Suppose that Assumption $H\left[f_{h}, g_{h}\right]$ and

1) $h \in H, \varphi_{h}: E_{h .0} \rightarrow \mathbb{R}$ is a given function and $u_{h}: E_{h .0} \cup E_{h} \rightarrow \mathbb{R}$ is the solution of the problem (8), (9),

2) the functions $v_{h}: E_{h .0} \cup E_{h} \rightarrow \mathbb{R}, \gamma, \alpha_{0}: H \rightarrow \mathbb{R}_{+}$are such that the conditions (22)-(24) are satisfied,

3) there is $c_{0} \in \mathbb{R}_{+}$such that the estimates

$$
\left|\delta_{i} v_{h}^{(r, m)}\right| \leqslant c_{0} \quad \text { on } E_{h}, \quad 1 \leqslant i \leqslant n,
$$

are satisfied. Then there exists a function $\alpha: H \rightarrow \mathbb{R}_{+}$such that

$$
\left|u_{h}^{(r, m)}-v_{h}^{(r, m)}\right| \leqslant \alpha(h) \quad \text { on } E_{h}
$$

and $\lim _{h \rightarrow 0} \alpha(h)=0$. 
Proof. Let the function $\Gamma_{h}: E_{h}^{\prime} \rightarrow \mathbb{R}$ be defined by the relation

$$
\delta_{0} v_{h}^{(r, m)}=F_{h}\left[v_{h}\right]^{(r, m)}+\Gamma_{h}^{(r, m)} .
$$

It follows from $(22)$ that $\left|\Gamma_{h}^{(r, m)}\right| \leqslant \gamma(h)$ on $E_{h}^{\prime}$. Let $J_{+}^{(r, m)}, J_{-}^{(r, m)}$ be the sets defined by (17), (18) and

$$
\begin{aligned}
\Lambda_{h}^{(r, m)}= & h_{0} \sum_{i \in J_{+}^{(r, m)}\left[u_{h}\right]} \frac{1}{h_{i}} f_{h . i}\left(t^{(r)}, x^{(m)}, u_{h}\right)\left(u_{h}-v_{h}\right)^{\left(r+1, m+e_{i}\right)} \\
& -h_{0} \sum_{i \in J_{-}^{(r, m)}\left[u_{h}\right]} \frac{1}{h_{i}} f_{h . i}\left(t^{(r)}, x^{(m)}, u_{h}\right)\left(u_{h}-v_{h}\right)^{\left(r+1, m-e_{i}\right)} .
\end{aligned}
$$

Since $u_{h}$ satisfies the difference problem (8), (9) then we have

$$
\begin{aligned}
\left(u_{h}-v_{h}\right)^{(r+1, m)}\left[1+h_{0} \sum_{i=1}^{n} \frac{1}{h_{i}}\left|f_{h . i}\left(t^{(r)}, x^{(m)}, u_{h}\right)\right|\right] \\
=\left(u_{h}-v_{h}\right)^{(r, m)}+\Lambda_{h}^{(r, m)} \\
\quad+h_{0} \sum_{i=1}^{n} \frac{1}{h_{i}}\left[f_{h . i}\left(t^{(r)}, x^{(m)}, u_{h}\right)-f_{h . i}\left(t^{(r)}, x^{(m)}, v_{h}\right)\right] \delta_{i} v_{h}^{(r+1, m)} \\
\quad+h_{0}\left[g_{h}\left(t^{(r)}, x^{(m)}, u_{h}\right)-g_{h}\left(t^{(r)}, x^{(m)}, v_{h}\right)\right]-h_{0} \Gamma_{h}^{(r, m)}
\end{aligned}
$$

Write $\varepsilon_{h}^{(r)}=\max \left\{\left|\left(u_{h}-v_{h}\right)^{(i, m)}\right|:\left(t^{(i)}, x^{(m)}\right) \in E_{h . r}\right\}$ for $0 \leqslant r \leqslant N_{0}$. It follows that

$$
\left|\Lambda_{h}^{(r, m)}\right| \leqslant h_{0} \varepsilon_{h}^{(r+1)} \sum_{i=1}^{n} \frac{1}{h_{i}}\left|f_{h . i}\left(t^{(r)}, x^{(m)}, u_{h}\right)\right|, \quad\left(t^{(r)}, x^{(m)}\right) \in E_{h}^{\prime} .
$$

We conclude from Assumption $H\left[f_{h}, g_{h}\right]$ and from (31), (34), (35) that the function $\varepsilon_{h}$ satisfies the recurrent inequality

$$
\varepsilon_{h}^{(r+1)} \leqslant \varepsilon_{h}^{(r)}+h_{0}\left(1+c_{0}\right) \sigma_{h}\left(t^{(r)}, \varepsilon_{h}^{(r)}\right)+h_{0} \gamma(h),
$$

where $0 \leqslant r \leqslant N_{0}-1$ and $\varepsilon_{h}^{(0)} \leqslant \alpha_{0}(h)$. Consider the solution $\eta_{h}: I_{h} \rightarrow \mathbb{R}_{+}$of the difference problem

$$
\begin{aligned}
\eta^{(r+1)} & =\eta^{(r)}+h_{0}\left(1+c_{0}\right) \sigma_{h}\left(t^{(r)}, \eta^{(r)}\right)+h_{0} \gamma(h), \quad 0 \leqslant r \leqslant N_{0}-1 \\
\eta^{(0)} & =\alpha_{0}(h) .
\end{aligned}
$$

It follows from the monotonicity of $\sigma_{h}$ and $(36)$ that $\varepsilon_{h}^{(r)} \leqslant \eta_{h}^{(r)}$ for $0 \leqslant r \leqslant N_{0}$. Then we obtain the assertion of Theorem 3.2 from the stability of the problem (25), (26). 


\section{Difference schemes for quasilinear equations}

We give examples of functions $f_{h}$ and $g_{h}$ corresponding to $f$ and $g$ and we prove that the implicit difference methods are convergent. We adopt additional assumptions for the mesh $E_{h .0} \cup E_{h}$. We assume that the steps of the mesh satisfy the condition: $h^{\prime}=M h_{0}$. Then we can write the definitions of the sets $E_{h .0}$ and $E_{h}$ in the following way:

$$
\begin{aligned}
E_{h .0} & =\left\{\left(t^{(r)}, x^{(m)}\right):-K_{0} \leqslant r \leqslant 0,-K \leqslant m \leqslant K\right\} \\
E_{h} & =\left\{\left(t^{(r)}, x^{(m)}\right): 0 \leqslant r \leqslant N_{0},\left|m_{r}\right| \leqslant K_{r}-r \text { for } r=1, \ldots, n\right\} .
\end{aligned}
$$

Assumption $H\left[T_{h}\right]$. There is an operator $T_{h}: F\left(E_{h .0} \cup E_{h}, \mathbb{R}\right) \rightarrow C\left(E_{0} \cup E, \mathbb{R}\right)$ such that

1) for $w, \tilde{w} \in F\left(E_{h .0} \cup E_{h}, \mathbb{R}\right)$ we have

$$
\left\|T_{h}[w]-T_{h}[\tilde{w}]\right\|_{t^{(r)}} \leqslant\|w-\tilde{w}\|_{h . r}, \quad 0 \leqslant r \leqslant N_{0},
$$

2) there is $\mu>0$ such that for each function $v: E_{h .0} \cup E_{h} \rightarrow \mathbb{R}$, which is of class $C^{2}$, there is $c_{0} \in \mathbb{R}_{+}$with the property

$$
\left\|v-T_{h}\left[v_{h}\right]\right\|_{t^{(r)}} \leqslant c_{0} h_{0}^{\mu}, \quad 0 \leqslant r \leqslant N_{0},
$$

where $v_{h}$ is the restriction of $v$ to the set $E_{h .0} \cup E_{h}$.

Remark 4.1. Condition (37) states that $T_{h}$ satisfies the Lipschitz condition with the constant $L=1$. It is clear that relation (37) implies the Volterra condition for $T_{h}$.

Assumption (38) means that the function $v$ is approximated by $T_{h}\left[v_{h}\right]$ and the error of this approximation is estimated by $c_{0} h_{0}^{\mu}$.

Remark 4.2. An example of the operator $T_{h}$ satisfying Assumption $H\left[T_{h}\right]$ is given in [8], see also [6]. Let $\tilde{C}$ be such a constant that for a function $v: E_{0} \cup E \rightarrow$ $\mathbb{R}$ we have

$$
\left|\partial_{t t} v(t, x)\right| \leqslant \tilde{C}, \quad\left|\partial_{t x_{j}} v(t, x)\right| \leqslant \tilde{C}, \quad\left|\partial_{x_{i} x_{j}} v(t, x)\right| \leqslant \tilde{C}, \quad i, j=1 \ldots, n,
$$

on $E_{0} \cup E$ and $C_{0}=\frac{\tilde{C}}{2}(1+\|M\|)^{2}$. The operator $T_{h}$ considered in [8] satisfies condition (38) for $\mu=2$ and for the above $C_{0}$.

Now we approximate solutions of the problem (1), (2) with solutions of the difference functional equation

$$
\delta_{0} z^{(r, m)}=\sum_{i=1}^{n} f_{i}\left(t^{(r)}, x^{(m)}, T_{h}[z]\right) \delta_{i} z^{(r+1, m)}+g\left(t^{(r)}, x^{(m)}, T_{h}[z]\right)
$$


with initial condition (9), where $\delta_{0}$ is defined by (5) and

$$
\begin{array}{ll}
\delta_{j} z^{(r, m)}=\frac{1}{h_{j}}\left[z^{\left(r, m+e_{j}\right)}-z^{(r, m)}\right] & \text { if } f\left(t^{(r)}, x^{(m)}, T_{h}[z]\right) \geqslant 0 \\
\delta_{j} z^{(r, m)}=\frac{1}{h_{j}}\left[z^{(r, m)}-z^{\left(r, m-e_{j}\right)}\right] & \text { if } f\left(t^{(r)}, x^{(m)}, T_{h}[z]\right)<0,
\end{array}
$$

where $1 \leqslant j \leqslant n$. Write

$$
\begin{aligned}
& E_{i}^{+}[\varepsilon]=\left\{(t, x) \in E: b_{i}-M_{i} t-\varepsilon<x_{i} \leqslant b_{i}-M_{i} t\right\} \\
& E_{i}^{-}[\varepsilon]=\left\{(t, x) \in E:-b_{i}+M_{i} t \leqslant x_{i}<-b_{i}+M_{i} t+\varepsilon\right\},
\end{aligned}
$$

where $1 \leqslant i \leqslant n$ and $\varepsilon>0$ satisfies the condition $\varepsilon<b_{i}-M_{i} a$ for $1 \leqslant i \leqslant n$.

Assumption $H[f, g]$. Suppose that

1) the functions $f: \Omega \rightarrow \mathbb{R}^{n}$ and $g: \Omega \rightarrow \mathbb{R}$ are continuous and they satisfy the Volterra condition,

2) there is $\sigma:[0, a] \times \mathbb{R}_{+} \rightarrow \mathbb{R}_{+}$such that

(i) $\sigma$ is continuous and it is nondecreasing with respect to both variables,

(ii) $\sigma(t, 0)=0$ for $t \in[0, a]$ and for each $c \geqslant 1$ the maximal solution of the Cauchy problem

$$
\omega^{\prime}(t)=c \sigma(t, \omega(t)), \quad \omega(0)=0,
$$

is $\tilde{\omega}(t)=0$ for $t \in[0, a]$,

3) the estimates

$$
\begin{aligned}
\|f(t, x, z)-f(t, x, \bar{z})\| & \leqslant \sigma\left(t,\|z-\bar{z}\|_{t}\right) \\
|g(t, x, z)-g(t, x, \bar{z})| & \leqslant \sigma\left(t,\|z-\bar{z}\|_{t}\right)
\end{aligned}
$$

are satisfied for $(t, x, z),(t, x, \bar{z}) \in \Omega$,

4) there is $\varepsilon>0$ such that

$$
\begin{array}{ll}
f_{i}(t, x, z) \leqslant 0 & \text { for }(t, x, z) \in E_{i}^{+}[\varepsilon] \times C\left(E_{0} \cup E, \mathbb{R}\right) \\
f_{i}(t, x, z) \geqslant 0 & \text { for }(t, x, z) \in E_{i}^{-}[\varepsilon] \times C\left(E_{0} \cup E, \mathbb{R}\right),
\end{array}
$$

where $1 \leqslant i \leqslant n$.

We give comments on the condition 4) of Assumption $H[f, g]$.

Remark 4.3. Suppose that Assumption $H[f, g]$ is satisfied and $z \in C\left(E_{0} \cup\right.$ $E, \mathbb{R})$. Let us consider the Cauchy problem

$$
\eta^{\prime}(\tau)=-f(\tau, \eta(\tau), z), \quad \eta(t)=x
$$

where $(t, x) \in E$. The solution $g[z](\cdot, t, x)=\left(g_{1}[z](\cdot, t, x), \ldots, g_{n}[z](\cdot, t, x)\right)$ of (42) is the bicharacteristic of equation (1) corresponding to $z$. Condition 4 ) of Assumption $H[f, g]$ states that 
(i) for each $(t, x) \in E_{i}^{+}[\varepsilon], 1 \leqslant i \leqslant n$, there is $\varepsilon_{0}>0$ such that the function $g_{i}[z](\cdot, t, x):\left[t-\varepsilon_{0}, t\right] \rightarrow \mathbb{R}$ is increasing,

(ii) for each $(t, x) \in E_{i}^{-}[\varepsilon], 1 \leqslant i \leqslant n$, there is $\varepsilon_{0}>0$ such that the function $g_{i}[z](\cdot, t, x):\left[t-\varepsilon_{0}, t\right] \rightarrow \mathbb{R}$ is decreasing.

This property of bicharacteristics is important in the construction of implicit difference schemes for $(1),(2)$. The difference operator $\left(\delta_{1}, \ldots, \delta_{n}\right)$ used in the paper satisfies the conditions:

(i) if the function $g_{i}[z](\cdot, t, x)$ is increasing on $\left[t-\varepsilon_{0}, t\right]$, then we put

$$
\delta_{i} z(t, x)=\frac{1}{\tau}\left[z(t, x)-z\left(t, x-\tau e_{i}\right)\right]
$$

where $1 \leqslant i \leqslant n$ and $\tau>0$,

(ii) if $g_{i}[z](\cdot, t, x)$ is decreasing on $\left[t-\varepsilon_{0}, t\right]$, then we put

$$
\delta_{i} z(t, x)=\frac{1}{\tau}\left[z\left(t, x+\tau e_{i}\right)-z(t, x)\right]
$$

where $1 \leqslant i \leqslant n$ and $\tau>0$.

Remark 4.4. Write

$$
\begin{aligned}
& \partial_{0} E_{i}^{+}=\left\{(t, x) \in E: x_{i}=b_{i}-M_{i} t\right\} \\
& \partial_{0} E_{i}^{-}=\left\{(t, x) \in E: x_{i}=-b_{i}+M_{i} t\right\},
\end{aligned}
$$

where $1 \leqslant i \leqslant n$. Suppose that there is $\widetilde{\varepsilon}>0$ such that

$$
\begin{array}{ll}
f_{i}(t, x, w)<-\widetilde{\varepsilon} & \text { for }(t, x, w) \in \partial_{0} E_{i}^{+} \times C\left(E_{0} \cup E, \mathbb{R}\right) \\
f_{i}(t, x, w)>\widetilde{\varepsilon} & \text { for }(t, x, w) \in \partial_{0} E_{i}^{-} \times C\left(E_{0} \cup E, \mathbb{R}\right),
\end{array}
$$

where $1 \leqslant i \leqslant n$. Then condition 4 ) of Assumption $H[f, g]$ is satisfied.

Remark 4.5. Suppose that the function $f: \Omega \rightarrow \mathbb{R}$ satisfies the condition

$$
x \diamond f(t, x, w) \leqslant \theta_{(n)} \quad \text { for }(t, x, w) \in \Omega,
$$

where $\theta_{(n)}=(0, \ldots, 0) \in \mathbb{R}^{n}$. Then condition 4) of Assumption $H[f, g]$ is satisfied.

Now we formulate the main result of the paper.

Theorem 4.6. Suppose that Assumptions $H\left[T_{h}\right]$ and $H[f, g]$ are satisfied and

1) $h \in H$, and there is a function $\alpha_{0}: H \rightarrow \mathbb{R}_{+}$such that

$$
\left|\varphi^{(r, m)}-\varphi_{h}^{(r, m)}\right| \leqslant \alpha_{0}(h) \text { on } E_{h .0} \quad \text { and } \quad \lim _{h \rightarrow 0} \alpha_{0}(h)=0
$$


2) $h^{\prime}=M h_{0}$ and the function $u_{h}: E_{h .0} \cup E_{h} \rightarrow \mathbb{R}$ is a solution of the problem (9), (39) with the operator $\delta$ defined by (40), (41),

3) the function $v: E_{0} \cup E \rightarrow \mathbb{R}$ is a solution of (1), (2) and $v$ is of class $C^{2}$, and $v_{h}$ is the restriction of function $v$ to the set $E_{h .0} \cup E_{h}$.

Then there is $\varepsilon>0$ and a function $\alpha: H \rightarrow \mathbb{R}_{+}$such that for $\|h\|<\varepsilon$ we have

$$
\left|u_{h}^{(r, m)}-v_{h}^{(r, m)}\right| \leqslant \alpha(h) \text { on } E_{h} \quad \text { and } \quad \lim _{h \rightarrow 0} \alpha(h)=0 .
$$

Proof. We prove that the functions $f_{h}(t, x, z)=f\left(t, x, T_{h}[z]\right)$ and $g_{h}(t, x, z)=$ $g\left(t, x, T_{h}[z]\right)$ satisfy all assumptions of the Theorem 3.1. First we show that the problem (25), (26) is stable in the sense of Assumption $H\left[\sigma_{h}\right]$. Let the functions $\alpha_{0}, \gamma: H \rightarrow \mathbb{R}_{+}$are such that $\lim _{h \rightarrow 0} \alpha_{0}(h)=0, \lim _{h \rightarrow 0} \gamma(h)=0$. Consider the solution $\eta_{h}: I_{h .0} \cup I_{h} \rightarrow \mathbb{R}_{+}$of the following difference problem:

$$
\begin{aligned}
\eta^{(r+1)} & =\eta^{(r)}+h_{0} c \sigma\left(t^{(r)}, \eta^{(r)}\right)+h_{0} \gamma(h), \quad 0 \leqslant r \leqslant N_{0}-1 \\
\eta^{(0)} & =\alpha_{0}(h) .
\end{aligned}
$$

Denote by $\omega_{h}:[0, a] \rightarrow \mathbb{R}_{+}$the maximal solution of the problem

$$
\omega^{\prime}(t)=c \sigma(t, \omega(t))+\gamma(h), \quad \omega(0)=\alpha_{0}(h) .
$$

There exists $\varepsilon>0$ such that the solution $\omega_{h}$ is defined on $[0, a]$ for $\|h\|<\varepsilon$ and $\lim _{h \rightarrow 0} \omega_{h}(t)=0$ uniformly on $[0, a]$. The function $\omega_{h}$ is convex on $[0, a]$, therefore it satisfies the difference inequality

$$
\omega_{h}^{(r+1, m)} \geqslant \omega_{h}^{(r)}+h_{0} c \sigma\left(t^{(r)}, \omega_{h}^{(r)}\right)+h_{0} \gamma(h), \quad 0 \leqslant r \leqslant N_{0}-1 .
$$

Since $\eta_{h}$ satisfies (46), (47), then we have $\eta_{h}^{(r)} \leqslant \omega_{h}^{(r)}$ for $0 \leqslant r \leqslant N_{0}$. This proves the stability of (25), (26). Moreover we have

$$
\begin{aligned}
\left\|f_{h}(t, x, z)-f_{h}(t, x, \bar{z})\right\| & =\left\|f\left(t, x, T_{h}[z]\right)-f\left(t, x, T_{h}[\bar{z}]\right)\right\| \\
& \leqslant \sigma\left(t,\left\|T_{h}[z-\bar{z}]\right\|_{t}\right) \\
& =\sigma\left(t,\|z-\bar{z}\|_{h . r}\right)
\end{aligned}
$$

and

$$
\left|g_{h}(t, x, z)-g_{h}(t, x, \bar{z})\right| \leqslant \sigma\left(t,\|z-\bar{z}\|_{h . r}\right) .
$$

Let us denote by $\widetilde{F}_{h}$ the Niemycki operator corresponding to (39), i.e.,

$$
\widetilde{F}_{h}[z]^{(r, m)}=\sum_{i=1}^{n} f_{i}\left(t^{(r)}, x^{(m)}, T_{h}[z]\right) \delta_{i} z^{(r+1, m)}+g\left(t^{(r)}, x^{(m)}, T_{h}[z]\right) .
$$

Consider the function $\widetilde{\Gamma}_{h}: E_{h}^{\prime} \rightarrow \mathbb{R}$ defined by the relation $\delta_{0} v_{h}^{(r, m)}=\widetilde{F}_{h}\left[v_{h}\right]^{(r, m)}+$ $\widetilde{\Gamma}_{h}^{(r, m)}$. We prove that there is $\widetilde{\gamma}: H \rightarrow \mathbb{R}_{+}$such that

$$
\left|\widetilde{\Gamma}_{h}^{(r, m)}\right| \leqslant \widetilde{\gamma}(h) \text { on } E_{h}^{\prime} \quad \text { and } \quad \lim _{h \rightarrow 0} \widetilde{\gamma}(h)=0 .
$$


Write

$$
\begin{aligned}
\Gamma_{h .0}^{(r, m)}= & \delta_{0} v_{h}^{(r, m)}-\partial_{t} v^{(r, m)}+\sum_{i=1}^{n} f_{i}\left(t^{(r)}, x^{(m)}, v\right)\left[\partial_{x_{i}} v^{(r, m)}-\delta_{i} v_{h}^{(r, m)}\right] \\
\Gamma_{h .1}^{(r, m)}= & \sum_{i=1}^{n}\left[f_{i}\left(t^{(r)}, x^{(m)}, v\right)-f_{i}\left(t^{(r)}, x^{(m)}, T_{h}\left[v_{h}\right]\right)\right] \delta_{i} v_{h}^{(r+1, m)} \\
& +g\left(t^{(r)}, x^{(m)}, v\right)-g\left(t^{(r)}, x^{(m)}, T_{h}\left[v_{h}\right]\right) .
\end{aligned}
$$

Then $\widetilde{\Gamma}_{h}^{(r, m)}=\Gamma_{h .0}^{(r, m)}+\Gamma_{h .1}^{(r, m)}$ on $E_{h}^{\prime}$. It follows easily that there is $\gamma_{0}: H \rightarrow \mathbb{R}_{+}$ such that $\left|\Gamma_{h .0}^{(r, m)}\right| \leqslant \gamma_{0}(h)$ on $E_{h}^{\prime}$ and $\lim _{h \rightarrow 0} \gamma_{0}(h)=0$. Suppose that $c_{0} \in \mathbb{R}_{+}$is defined by the relations $\left|\partial_{x_{i}} v(t, x)\right| \leqslant c_{0},(t, x) \in E, 1 \leqslant i \leqslant n$. It follows from Assumptions $H[f, g]$ and $H\left[T_{h}\right]$ that there is $\bar{c} \in \mathbb{R}_{+}$such that

$$
\left|\Gamma_{h .1}^{(r, m)}\right| \leqslant\left(1+c_{0}\right) \sigma\left(t^{(r)}, \quad\left\|v-T_{h}\left[v_{h}\right]\right\|_{t^{(r)}}\right) \leqslant\left(1+c_{0}\right) \sigma\left(a, \bar{c} h_{0}^{\mu}\right) .
$$

Then condition (48) is satisfied with $\widetilde{\gamma}(h)=\gamma_{0}(h)+\left(1+c_{0}\right) \sigma\left(a, \bar{c} h_{0}^{\mu}\right)$. The assertion of Theorem 4.6 then follows from Theorem 3.2.

Remark 4.7. Suppose that Assumption $H[f, g]$ is satisfied with $\sigma(t, \xi)=L \xi$, $(t, \xi) \in[0, a] \times \mathbb{R}_{+}$, where $L \in \mathbb{R}_{+}$. Then we have assumed that $f$ and $g$ satisfy the Lipschitz condition with respect to the functional variable. In this case we have the estimates

$$
\left|u_{h}^{(r, m)}-v_{h}^{(r, m)}\right| \leqslant \begin{cases}\alpha_{0}(h) e^{c L a}+\gamma(h) \frac{e^{c L a}-1}{c L} & \text { if } L>0 \\ \alpha_{0}(h)+\gamma(h) a & \text { if } L=0 .\end{cases}
$$

The above estimates are obtained by solving problem (46), (47).

\section{Solutions of implicit difference equations on rectangular domains}

Suppose that Assumption $H[f, g]$ is satisfied with $\Omega=E \times C\left(E_{0} \cup E, \mathbb{R}\right)$ where $E=[0, a] \times[-b, b]$. Assume also that condition (43) is satisfied. Then the natural domain of the existence of solutions to problem (1), (2) is the set $E_{0} \cup([0, a] \times$ $[-b, b])$. We give a simple method for solving of the implicit difference problem (9), (39) in this case.

Suppose that $0 \leqslant r \leqslant N_{0}$ is fixed and that solution $u_{h}$ of (9), (39) is defined on the set $E_{h . r}$. We first compute $z_{h}^{(r+1, m)}$ for $\theta_{(n)} \leqslant m \leqslant K$. According to assumption (43) we have

$$
f_{i}\left(t^{(r)}, x^{(m)}, T_{h}\left[z_{h}\right]\right) \leqslant 0, \quad 1 \leqslant i \leqslant n
$$


where $0 \leqslant r<N_{0}$ and $\theta_{(n)} \leqslant m \leqslant K$, and

$$
f_{i}\left(t^{(r)}, x^{(m)}, T_{h}\left[z_{h}\right]\right)=0, \quad 1 \leqslant i \leqslant n
$$

for such $m=\left(m_{1}, \ldots, m_{n}\right)$ that $m_{i}=0$. We conclude from (40), (41) that equation (39) for $\theta_{(n)} \leqslant m \leqslant K$ has the form

$$
\begin{aligned}
& z_{h}^{(r+1, m)}\left[1-h_{0} \sum_{i=1}^{n} \frac{1}{h_{i}} f_{i}\left(t^{(r)}, x^{(m)}, T_{h}\left[z_{h}\right]\right)\right] \\
& =-h_{0} \sum_{i=1}^{n} \frac{1}{h_{i}} f_{i}\left(t^{(r)}, x^{(m)}, T_{h}\left[z_{h}\right]\right) z_{h}^{\left(r+1, m-e_{i}\right)}+z_{h}^{(r, m)}+h_{0} g\left(t^{(r)}, x^{(m)}, T_{h}\left[z_{h}\right]\right)
\end{aligned}
$$

We deduce from (49) that $z_{h}^{(r+1, m)}$ may be computed for $m=\theta_{(n)}$ and for $m=\left(m_{1}, 0, \ldots, 0\right) \in Z^{n}$ where $1 \leqslant m_{1} \leqslant K_{1}$. Our next goal is to determine $z_{h}^{(r+1, m)}$ for $m=\left(m_{1}, m_{2}, 0, \ldots, 0\right) \in Z^{n}$ where $0 \leqslant m_{1} \leqslant K_{1}, 0 \leqslant m_{2} \leqslant K_{2}$. We conclude from (49) that the numbers $z_{h}^{(r+1, m)}$ for the above $m$ exist and that they are unique.

Suppose that the solution $z_{h}^{(r+1, m)}$ is computed for $m=\left(m_{1}, \ldots, m_{\kappa}, 0, \ldots, 0\right)$ $\in Z^{n}$ where $0 \leqslant m_{i} \leqslant K_{i}$ and $1 \leqslant \kappa \leqslant n-1$ is fixed. Repeated application of (49) enables us to calculate $z_{h}^{(r+1, m)}$ for $m=\left(m_{1}, \ldots, m_{\kappa+1}, 0, \ldots, 0\right) \in Z^{n}$ where $0 \leqslant m_{i} \leqslant K_{i}$ and $1 \leqslant i \leqslant \kappa+1$. It follows from the above considerations that the solution $z_{h}^{(r+1, m)}$ of (9), (39) may be calculated for $\theta_{(n)} \leqslant m \leqslant K$.

In a similar way the solution $z_{h}^{(r+1, m)}$ may be computed on the set $E_{h . r+1} \cap$ $\left(X_{1} \times X_{2} \times \ldots \times X_{n}\right)$ where $X_{i}=\mathbb{R}_{+}$or $X_{i}=R_{-}, R_{-}=(-\infty, 0]$. Then our problem is solved by induction with respect to $r, 0 \leqslant r \leqslant N_{0}$.

Remark 5.1. It is easy to see that condition (43) may be replaced by the following assumption: there exists $\widetilde{x},-b<\widetilde{x}<b$ such that $(x-\widetilde{x}) \diamond f(t, x, w) \leqslant$ $\theta_{(n)}$ for $(t, x, w) \in \Omega$.

\section{Numerical examples}

We give now numerical examples concerning our theory for quasilinear differential equations. To find an approximate solutions we use implicit difference method of the Euler type and explicit difference method with difference operators given by the Lax scheme.

Example 6.1. For $n=1$ we put

$$
E=\{(t, x): t \in[0,0.5], \quad x \in[-1,1]\} .
$$


Consider the differential equation

$$
\begin{aligned}
\partial_{t} z(t, x)= & -x\left[1+\cos \left(z(t, 0.5 x)-e^{t x}\right)\right] \partial_{x} z(t, x) \\
& +\sin \left(z(t,-0.5 x)-e^{-t x}\right)+2 x(1+2 t) z(t, x)
\end{aligned}
$$

with the initial condition

$$
z(0, x)=1, \quad x \in[-1,1] .
$$

Note that condition (43) is satisfied for equation (51). The exact solution of this problem is known. It is $v(t, x)=e^{2 t x}$. Let $h=\left(h_{0}, h_{1}\right)$, stand for steps of the mesh on E.

Let us denote by $u_{h}: E_{h} \rightarrow \mathbb{R}$ the solution of the implicit difference problem corresponding to (51), (52). Write

$$
\eta_{h}^{(r)}=\frac{1}{2 K+1} \sum_{m=-K}^{K}\left|u_{h}^{(r, m)}-v^{(r, m)}\right|,
$$

where $K \in \mathbb{N}$ is defined by the condition $K h_{1}=1$. The numbers $\eta_{h}^{(r)}$ are the arithmetical mean of the errors with fixed $t^{(r)}$. The values of the function $\eta_{h}$ are listed in Table 1.

\begin{tabular}{c|c|c} 
& $h=\left(10^{-2}, 10^{-3}\right)$ & $h=\left(2 \cdot 10^{-3}, 2 \cdot 10^{-4}\right)$ \\
\hline$t=0.1$ & 0.001842 & 0.000371 \\
$t=0.2$ & 0.003532 & 0.000714 \\
$t=0.3$ & 0.005214 & 0.001055 \\
$t=0.4$ & 0.006989 & 0.001416 \\
$t=0.5$ & 0.008937 & 0.001813
\end{tabular}

Table 1: The error $\eta_{h}$.

The results shown in Table 1 are consistent with our mathematical analysis. We consider also an approximate solution $z_{h}$ of $(51),(52)$ which is obtained by using the classical Lax scheme. The domain of $z_{h}$ is the set

$$
\widetilde{E}_{h}=\left\{\left(t^{(r)}, x^{(m)}\right): 0 \leqslant r \leqslant N_{0}, x^{(m)} \in\left[-1+t^{(r)}, 1-t^{(r)}\right]\right\}
$$

and $N_{0} h_{0} \leqslant 0.5<\left(N_{0}+1\right) h_{0}$. Write

$$
\widetilde{\eta}_{h}=\max \left\{\left|z_{h}^{(r, m)}-v^{(r, m)}\right|:\left(t^{(r)}, x^{(m)}\right) \in \widetilde{E}_{h}\right\} .
$$

In the considered cases the values of $\widetilde{\eta}_{h}$ are bigger than $10^{2}$. 
Example 6.2. Let $E \subset R^{2}$ be defined by (50). Consider the differential integral equation

$$
\begin{aligned}
\partial_{t} z(t, x)= & -2 \sin x\left[1+\sin \left(3 \int_{-x}^{x} z(t, s) d s-2 x z(t, x)\right)\right] \partial_{x} z(t, x) \\
& +\int_{0}^{t} z(\tau, x) d \tau+x^{2}(1+t)+4 x\left(e^{t}-1\right) \sin x
\end{aligned}
$$

with the initial condition

$$
z(0, x)=0, \quad x \in[-1,1] .
$$

Note that equation (56) satisfies (43). The exact solution of this problem is known. It is $v(t, x)=\left(e^{t}-1\right) x^{2}$. Put $h=\left(h_{0}, h_{1}\right)$ stand for the steps of the mesh on E.

Let us denote by $u_{h}: E_{h} \rightarrow \mathbb{R}$ the solution of the implicit difference problem corresponding to (56), (57). Let $\eta_{h}$ be defined by (53). The numbers $\eta_{h}^{(r)}$ are the arithmetical mean of the errors with fixed $t^{(r)}$. The values of the function $\eta_{h}$ are listed in Table 2.

\begin{tabular}{c|c|c} 
& $h=\left(10^{-2}, 10^{-3}\right)$ & $h=\left(2 \cdot 10^{-3}, 2 \cdot 10^{-4}\right)$ \\
\hline$t=0.1$ & 0.001466 & 0.002330 \\
$t=0.2$ & 0.003891 & 0.002330 \\
$t=0.3$ & 0.007605 & 0.002330 \\
$t=0.4$ & 0.012503 & 0.010204 \\
$t=0.5$ & 0.018385 & 0.016133
\end{tabular}

Table 2: The error $\eta_{h}$.

The results given in Table 2 are consistent with our theoretical results. We consider also an approximate solution $z_{h}$ of (56),(57) which is obtained by using the classical Lax scheme. The domain of $z_{h}$ is the set $\widetilde{E}_{h}$ defined by $(54)$. Let $\widetilde{\eta}_{h}$ be defined by (55). In the considered cases the values of $\widetilde{\eta}_{h}$ are bigger than $10^{2}$.

The above examples show that there are implicit difference schemes for problem (1), (2) which are convergent and the corresponding classical difference methods are not convergent. This is due to the fact that we need the (CFL) conditions in the classical case and we do not need special assumptions on the steps of the mesh in the case of implicit difference schemes.

Note that there is a natural class of differential equations (1) for which the implicit difference problems can be easily solved. This class of equations is described in Section 5.

\section{References}

[1] Baranowska, A. and Kamont, Z., Numerical method of lines for first order partial differential functional equations. Z. Anal. Anwendungen 21 (2002), $949-962$. 
[2] Brandi, P., Kamont, Z. and Salvadori, A., Difference methods for nonlinear first order partial differential equations with mixed boundary conditions. Math. Balkanica 10 (1996)(2-3), 249 - 269.

[3] Brunner, H., The numerical treatment of ordinary and partial Volterra integrodifferential equations. In: Proc. First Internat. Colloq. Numer. Anal. (Plovdiv 1992; eds.: D. Bainov et al.). Utrecht: VSP 1993, pp. 13 - 26.

[4] Godlewski, E., Raviart, P. A., Numerical Approximation of Hyperbolic Systems of Conservation Laws. Berlin: Springer 1996.

[5] Ivanauskas, F. F., On solutions of the Cauchy problem for a system of differential integral equations (in Russian). Zh. Vychisl. Mat. i Mat. Fiz. 18 (1978), $1025-1028$.

[6] Jaruszewska-Walczak, D. and Kamont, Z., Difference methods for quasilinear hyperbolic differential functional systems on the Haar pyramid. Bull. Belg. Math. Soc. Simon Stevin 10 (2003)(2), $267-290$.

[7] Jaruszewska-Walczak, D. and Kamont, Z., Numerical methods for hyperbolic functional differential equations on the Haar pyramid. Computing 65 (2000), $45-72$.

[8] Kamont, Z., Hyperbolic Functional Differential Inequalities and Applications. Dordrecht: Kluwer 1999.

[9] Kępczyńska, A., Implicit difference methods for first order partial differential functional equations. Nelin. Koliv. 8 (2005), 201 - 215; English transl.: Nonlinear Oscil. 8 (2005), $201-215$.

[10] Kępczyńska, A., Implicit difference methods for nonlinear first order partial differential equations. Univ. Jagiel. Acta Math. 43 (2005), 143 - 164.

[11] Kępczyńska, A., Implicit difference functional inequalities and applications. Math. Balk. (to appear).

[12] Lakshmikantham, V. and Leela, S., Differential and Integral Inequalities. New York: Academic Press 1969.

[13] Leszczyński, H., Convergence results for unbounded solutions of first order nonlinear differential functional equations. Ann. Polon. Math. 64(1996), 1 - 16.

[14] Prządka, K., Difference methods for nonlinear partial differential functional equations of the first order. Math. Nachr. 138 (1988), $105-123$.

[15] Szarski, J., Differential Inequalities. Warszawa: Polish Sci. Publ. 1967.

[16] Tran Due Van, Mikio Tsui and Nguyen Duy Thai Son, The Characteristic Method and its Applications for First-Order Nonlinear Partial Differential Equations. Boca Raton: Chapman \& Hall 2000.

Received January 30, 2006 